\title{
In-Vitro Activity of Teicoplanin against Clinical Methicillin Resistant Staphylococcus aureus Isolates
}

\author{
Ravi T1, Deepak $\mathrm{K}^{1,2^{*}}$, Nayak J1, Manish Kumar P ${ }^{2}$ and Prakash V² \\ 1Department of Applied Microbiology, Banaras Hindu University, India \\ ${ }^{2}$ Department of Microbiology, Banaras Hindu University, India
}

\section{Research Article}

Volume 2 Issue 1

Received Date: February 08, 2017

Published Date: March 02, 2017

DOI: $10.23880 /$ oajmb-16000112

*Corresponding author: Dr. Deepak Kumar, Assistant Professor, Department of Microbiology, Institute of Medical Sciences, Banaras Hindu University, Varanasi-221005, India, Tel: +91-9451354332; E-mail: deepak.mln30@gmail.com

Abstract

Aim: To evaluate the in vitro activity of teicoplanin against clinical methicillin resistant Staphylococcus aureus isolates was the aim of this study

Methods and Material: A total of 62 previously confirmed MRSA isolates were included in this study. Susceptibility testing and result interpretation of isolates to teicoplanin was performed by the E test and disk diffusion method as per Clinical and Laboratory Standards Institute (CLSI) M100-S25 recommendations.

Results: Teicoplanin appears to exhibit excellent in vitro activity against the MRSA isolates.

Conclusion: The high in vitro susceptibility to teicoplanin in this population and fewer adverse drug effects, teicoplanin may be considered a useful first line antibiotic agent in the treatment of infections caused by MRSA.

Keywords: Teicoplanin; MRSA; E Test; Disk Diffusion Method

\section{Introduction}

Methicillin-resistant Staphylococcus aureus (MRSA) are among the most prevalent pathogen responsible for hospital acquired infections [1]. MRSA infection is hard to treat as methicillin resistance in general is associated with other class of drugs like macrolides, lincosamides, aminoglycosides, quinolones, and trimethoprimsulfamethoxazole. The treatment option for this multidrug resistance organism is limited to glycopeptide antibiotics like vancomycin and teicoplanin. Vancomycin usage is presently a concern to clinician because of decreased efficacy despite MIC value in susceptible range [2]. Other factors restricting its use are MIC creep phenomenon and side effects like ototoxicity, and nephrotoxicity [3]. Teicoplanin on the other hand hold the promise to be a useful alternative in cases where vancomycin is clinically ineffective and in cases where use of vancomycin is curtailed due to adverse drug affects $[4,5]$.

Teicoplanin is a naturally occurring lipoglycopeptide that kills Gram-positive cocci by inhibiting peptidoglycan biosynthesis by binding to the D-alanyl-D-alanine termini of peptidoglycan intermediates, thus disrupting late-stage steps in cell wall biosynthesis [6]. This drug shows a great therapeutic effect to MRSA. With the constant change in the epidemiology of MRSA throughout regions and countries a variation in its drug-resistance patterns is reported [7]. Unfortunately no current resistance data from our area are available. It is thus mandatory to monitor the trends of resistance to teicoplanin in the MRSA causing infection. The aim of this study was evaluate the in vitro teicoplanin activity against clinical MRSA isolates. 


\section{Material and Methods}

The study was carried out in the Department of Microbiology, Institute of Medical Science Banaras Hindu University, Varanasi U.P. India. The total duration of study is one year; period extends from July 2015 to June 2016. A total of 62 non-duplicate MRSA isolates from various clinical specimen like pus, blood, urine, tracheal aspirates, sputum, central venous catheters tips, CSF and other sterile body fluids were randomly selected. MRSA isolates were identified by standard microbiological techniques. Methicillin resistance was confirmed by using cephoxitin disc $(30 \mu \mathrm{g}$ Himedia labs) After isolation and identification, the MRSA strains were kept at $-20^{\circ} \mathrm{C}$ in peptone/glycerol $(30 \% \mathrm{w} / \mathrm{v})$, and before teicoplanin susceptibility testing, the strains were purified twice on blood agar plates.

Susceptibility testing of the isolates to teicoplanin was performed by the disk diffusion method as per Clinical and Laboratory Standards Institute (CLSI M100-S25) [8]. The MIC values were obtained by E-test, which was performed as per manufacturer's instructions using gradient strips of teicoplanin (Biomerieux France). The disc diffusion test was performed with cation adjusted Mueller-Hinton agar (Himedia Labs) as testing media. Teicoplanin disks of content $30 \mu \mathrm{g}$ (BD USA) was used. The inoculated plates were incubated in ambient air at $37^{\circ} \mathrm{C}$ for 16 to $18 \mathrm{~h}$. MRSA strain ATCC 43300 was used as control strains, zone of inhibition and MIC values obtained was interpreted by using CLSI M100-S25 (2015) breakpoints. The isolate was considered susceptible to teicoplanin if the zone of inhibition $\geq 14 \mathrm{~mm}$; intermediate if zone of inhibition $\leq 10 \mathrm{~mm}$, and intermediate if zone size is in range $11-13 \mathrm{~mm}$. The MIC breakpoints for teicoplanin as pre CLSI was ( $<8 \mu \mathrm{g} / \mathrm{ml}, \mathrm{S} ; 16 \mu \mathrm{g} / \mathrm{ml}, \mathrm{I}$; $>32 \mu \mathrm{g} / \mathrm{ml}, \mathrm{R}$ ).

\section{Results}

A total of 62 clinical MRSA isolates were included in this study. Table: 1 shows distribution of MRSA among various clinical samples. From the above table it can be said the highest number of methicillin resistant strains were obtained from pus (38.7\%) followed by blood (16.1 $\%)$ and sputum (12.9\%).

The main finding of our study is that teicoplanin exhibits excellent antimicrobial activity against MRSA isolates as none of the isolates obtained was resistant to teicoplanin. The distribution of zone of inhibition is soon in table 2. The zone size of inhibition by disc-diffusion method ranges from $14-18 \mathrm{~mm}$ with median value of
$15.7 \mathrm{~mm}$. Majority of the isolate $(53.2 \%)$ had zone of inhibition $>16 \mathrm{~mm}$. The distribution of MIC values and MIC range for teicoplanin is shown in table 3. The MIC values obtained by E-test ranges from $0.38-2.00 \mu \mathrm{g} / \mathrm{ml}$ with a mean value of $1.00 \mu \mathrm{g} / \mathrm{ml}$. For majority of isolate $(69.3 \%)$ the MIC values were $<1 \mu \mathrm{g} / \mathrm{ml}$. After applying the CLSI interpretative criteria all the MRSA isolates were found susceptible to teicoplanin by both the methodology. Thus a $100 \%$ concordance between the results of E-test and DD method was found.

\begin{tabular}{|c|c|c|c|}
\hline S. no & Clinical specimens & $\begin{array}{c}\text { Number of } \\
\text { isolates (n) }\end{array}$ & $\begin{array}{c}\text { Percentage } \\
\text { (\%) }\end{array}$ \\
\hline 1 & Pus & 24 & 38.7 \\
\hline 2 & Blood & 10 & 16.1 \\
\hline 3 & Urine & 3 & 4.8 \\
\hline 4 & Sputum & 8 & 12.9 \\
\hline 5 & Tracheal aspirates & 5 & 8.1 \\
\hline 6 & $\begin{array}{c}\text { Central venous } \\
\text { catheters tips }\end{array}$ & 2 & 3.2 \\
\hline 7 & CSF & 1 & 1.6 \\
\hline 8 & $\begin{array}{c}\text { Other Sterile body } \\
\text { fluids }\end{array}$ & 9 & 14.5 \\
\hline & Total & 62 & 100 \\
\hline
\end{tabular}

Table 1: Distribution of MRSA isolates from various clinical specimens.

\begin{tabular}{|c|c|c|}
\hline Zone diameter (mm) & Number(n) & Percentage (\%) \\
\hline$>\mathbf{1 6}$ & 33 & 53.2 \\
\hline $\mathbf{1 4 - 1 5}$ & 29 & 46.8 \\
\hline $\mathbf{1 1 - 1 3}$ & 0 & 0 \\
\hline$<\mathbf{1 0}$ & 0 & 0 \\
\hline
\end{tabular}

Table 2: Distribution of zone of inhibition for teicoplanin for 62 MRSA isolates.

\begin{tabular}{|c|c|c|}
\hline MIC $(\boldsymbol{\mu g} / \mathbf{m l})$ & Number(n) & Percentage (\%) \\
\hline$<\mathbf{0 . 0 5}$ & 11 & 17.7 \\
\hline $\mathbf{0 . 7 5 - 1 . 0}$ & 32 & 51.6 \\
\hline $\mathbf{1 . 5 - 2 . 0}$ & 19 & 30.7 \\
\hline$>\mathbf{4}$ & 0 & 0 \\
\hline
\end{tabular}

Table 3: Distribution of MIC values for teicoplanin for 62 MRSA isolates.

\section{Discussion}

Methicillin-resistant Staphylococcus aureus (MRSA) is causing a wide variety of human diseases and hence increasing burden on healthcare resources. Vancomycin has been the only uniformly effective treatment for MRSA infections in India, and other glycopeptides not being 


\section{Open Access Journal of Microbiology \& Biotechnology}

commonly used. The role of vancomycin in the treatment of MRSA has been questioned and debated due to the spread of vancomycin-intermediate $S$. aureus (VISA), and vancomycin-resistant $S$. aureus (VRSA) $[9,10]$. Teicoplanin, a glycopeptide class antibiotic is used intravenously or intramuscular to treat serious MRSA infections. In our study, we examined the susceptibility of teicoplanin by disc-diffusion and E-test. Few studies have reported that detection of teicoplanin resistance by conventional disc diffusion methods is difficult because of limited diffusion of its large molecule in agar [11]. This study did not find and limitations of disc susceptibility testing for MRSA with teicoplanin. Both methods provided similar results and we did not find any resistance to teicoplanin in our tested MRSA isolates.

The data presented in this study support the findings of studies performed worldwide which says that the number of MRSA resistant to teicoplanin is very low [12]. Several studies have demonstrated the relationship between teicoplanin MICs values and treatment outcome in patients with MRSA infections. A study by Charlesworth et al showed that a higher teicoplanin MIC was associated with a lower survival rate in critically ill patients [13]. The present study confirms the good in vitro activity of teicoplanin against MRSA as the mean MIC values of teicoplanin in our study was $1.00 \mathrm{mg} / \mathrm{L}$. The high in vitro susceptibility of the isolates tested to teicoplanin and low resistance prevalence may be related to its restricted use for the treatment of MRSA infections. Teicoplanin thus, may be considered a promising treatment option for the treatment of MRSA infections in our region.

\section{Conclusion}

Based on the above results, it can be concluded that teicoplanin, is a good choice for the treatment against bacterial infections caused by methicillin resistant Staphylococcus aureus (MRSA). The comparative analysis demonstrates that disc-diffusion and E-test provide similar results of susceptibility. Thus, for susceptibility testing disc diffusion can be easier and economical option as compared to E test.

\section{Acknowledgement}

The authors would like to thanks Mr. Atul Kumar Awasthi area manager SANOFI for providing Teicoplanin Disc and E strips for this study.

\section{References}

1. Boyce JM, Cookson B, Christiansen K, Hori S, Vuopio VJ, et al. (2005) Methicillin-resistant Staphylococcus aureus. Lancet Infect Dis 5(10): 653-663.

2. Lodise TP, Graves J, Evans A, Graffunder E, Helmecke $M$, et al. (2008) Relationship between vancomycin MIC and failure among patients with methicillinresistant Staphylococcus aureus bacteremia treated with vancomycin. Antimicrob Agents Chemother 52(9): 3315-3320.

3. Steinkraus G, White R, Friedrich L (2007) Vancomycin MIC creep in non vancomycin- intermediate Staphylococcus aureus (VISA), vancomycin susceptible clinical methicillin-resistant $\mathrm{S}$. aureus (MRSA) blood isolates from 2001-05. J Antimicrob Chemother 60(4): 788-794.

4. Marinho DS, Huf G, Ferreira BL, Castro H, Rodrigues CR, et al. (2011) The study of vancomycin use and its adverse reactions associated to patients of a brazilian university hospital. BMC Res Notes 4: 236.

5. Cavalcanti $A B$, Goncalves AR, Almeida CS, Bugano DD, Silva E (2010) Teicoplanin versus Vancomycin for proven or suspected infection. Cochrane Database Syst Rev 16(6).

6. Svetitsky S, Leibovici L, Paul M (2009) Comparative efficacy and safety of vancomycin versus teicoplanin: systematic review and meta-analysis. Antimicrob Agents Chemother 53(10): 4069-4079.

7. Rodríguez NE, Seas C, Guzmán BM, Mejía C, Alvarez C, et al. (2010) Evolution of methicillin-resistant Staphylococcus aureus clones in Latin America. Int J Infect Dis 14(7): 560-566.

8. Clinical and Laboratory Standards Institute (2015) Performance Standards for Antimicrobial Susceptibility Testing; Twenty-Fifth Informational Supplement. CLSI document.

9. Hiramatsu K, Aritaka N, Hanaki H, Kawasaki S, Hosoda Y, et al. (1997) Dissemination in Japanese hospitals of strains of Staphylococcus aureus heterogeneously resistant to vancomycin. Lancet 350 : 1670-1673. 
10. Hiramatsu K (2001) Vancomycin-resistant Staphylococcus aureus: a new model of antibiotic resistance. Lancet Infect Dis 1(3): 147-155.

11. Cavenaghi LA, Biganzoli E, Danese A, Parenti F (1992) Diffusion of teicoplanin and vancomycin in agar. Diagn Microbiol Infect Dis 15(3): 253-258.

12. Samra Z, Ofer O, Shmuely H (2005) Susceptibility of methicillin-resistant Staphylococcus aureus to vancomycin, teicoplanin, linezolid, pristinamycin and other antibiotics. Isr Med Assoc J 7(3): 148-150.

13. Charlesworth $\mathrm{R}$, Warner $\mathrm{M}$, Livermore DM, Wilson AP (2006) Comparison of four methods for detection of teicoplanin resistance in methicillin-resistant Staphylococcus aureus. J Antimicrob Chemother 58: 186-189.

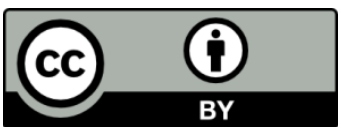

